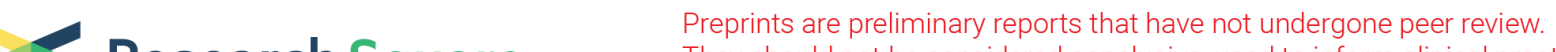 Research Square
They should not be considered conclusive, used to inform clinical practice,
or referenced by the media as validated information.
}

\section{Multicenter study of pediatric alimentary tract magnetic foreign body ingestion in China}

\section{Kai Wang}

Beijing Children's Hospital

\section{Dan Zhang}

Beijing Children's Hospital

\section{Xianling Li}

Beijing Children's Hospital

\section{Zengmeng Wang}

Beijing Children's Hospital

Guangjun Hou

Zhengzhou Children's Hospital

\section{Xinjian Jia}

Xi'an Children's Hospital

Huizhong Niu

Children's Hospital of Hebei Province

\section{Shiqin Qi}

Anhui Provincial Children's Hospital

\section{Qingqiang Deng}

Jiangxi Provincial Children's Hospital

\section{Bin Jiang}

Children's Hospital of Nanjing Medical University

\section{Hongqiang Bian}

Wuhan Children's Hospital

\section{Heying Yang}

the First Affiliated Hospital of Zhengzhou University

\section{Yajun Chen ( $\nabla$ chenyajun@bch.com.cn )}

Beijing Children's Hospital, Capital Medical University, National Center for Children's Health https://orcid.org/0000-0001-9103-5054

\section{Research article}

Keywords: Pediatric, Alimentary tract, Magnetic foreign body, Buckball

Posted Date: August 28th, 2019 
DOI: https://doi.org/10.21203/rs.2.10646/v1

License: (c) (1) This work is licensed under a Creative Commons Attribution 4.0 International License. Read Full License 


\section{Abstract}

Abstract Background: To investigate the alimentary tract Buckyball injuries in Chinese pediatric patients, and describe the managements and precautionary measures through multicenter investigation. Methods: Medical records of 74 pediatric patients from 9 large Chinese third-grade class-A hospitals were included in this study. Questionnaires were distributed online, and medical records were reviewed. Follow-ups were through telephone and outpatient service. Results: Among the 74 cases were 50 boys (68\%) and 24 girls $(32 \%)$. The age was $36.00(21.75,77.00)$ months, range from 7 months to 11 years old, and it showed two peaks, between one and three years old, and from six to eleven years old. The annual case numbers showed sharp increase, and Hebei Province had the largest number of 23 cases. Only 3 of them had exact reasons for swallowing the balls, the rest 71 patients had no intension for the ingestion. From onset to the emergency department time was $2.00(1.00,5.25)$ days, varied from 4 hours to 40 days. Twentyone patients had no symptoms, and abdominal pain, vomiting, fever, abdominal distension, fiercely crying, melena, and flatus and defecation stopped were in 40 (54.05\%), 36 (48.65\%), 11 (14.86\%), 3 (4.05\%), 3 (4.05\%), 2 (2.70\%), 1 (1.35\%) cases. Gastroscope, coloscope, laparoscopic operation, and laparotomy operation were adopted. Gastrointestinal perforation, ischemia and necrosis of gastrointestinal wall, abscess, ileus, fistula was found in 38 (51.53\%), 14 (18.92\%), 7 (9.46\%), 6 (8.11\%), 2 $(2.70 \%)$, other $28(37.84 \%)$ patients had gastrointestinal mucosa erosion. The numbers of Buckyball ingested was $4(2,8)$, with a range from 1 to 39 . During the follow-up period of $6(1,15)$ months, three patients had ileus, and one suffered a second operation. The remaining 71 patients were uneventful. None of the 74 patients reported a second time swallowing of Buckyballs or other magnetic foreign bodies. Conclusions: Pediatric alimentary tract magnetic foreign body ingestion in China is still increasing. Treatments and managements are of great significance, while prevention is more important and time critical. Precautionary measures toward the issue should be taken from three levels. Keywords: Pediatric, Alimentary tract, Magnetic foreign body, Buckball

\section{Introduction}

Alimentary tract foreign body ingestion is common in children, especially in neonates and infants [1]. Researches showed that $80 \%$ of the ingestions would cause no harm, and the foreign body would pass out of alimentary tract without incident [2]. While button batteries and magnetic foreign bodies are special among them, as they could lead to severer injuries to the gastrointestinal tract, or even be lifethreatening. Unlike button batteries, which could form local micro electrical current and cause chemical erosion to the gastrointestinal wall, magnetic foreign bodies have another pathogenesis. When it is swallowed alone, or multi magnetic foreign bodies are swallowed at the same time, they tend to discharge spontaneously. However, once they are ingested separately, or they are ingested separately with metallic foreign body, they could attract each other through the alimentary tract, and cause ischemia, necrosis, perforation and volvulus of the intestinal wall, leading to severe onset.

Buckyballs, an extraordinary kind of toy with the same size of $5 \mathrm{~mm}$ in diameter, made of rare-earth magnets, also named magic magnetic balls, are becoming more and more popular in China recently, and 
the emergency department has more encounters with pediatric gastrointestinal Buckyball injuries along with the popularity. Unlike the traditional magnets, Buckyball is small in volume, while powerful in magnetism. Since now, no large-scale case study has concluded the severity of gastrointestinal Buckyball injuries in Chinese pediatric patients.

Thus, the purpose of the study is to investigate the alimentary tract Buckyball injuries in Chinese pediatric patients, and describe the managements and precautionary measures toward the issue through multicenter investigation.

\section{Methods}

\section{Patients}

Medical records of 74 pediatric patients, under the age of 18 years old, diagnosed as alimentary tract Buckyball ingestion, admitted to emergency department, undertook endoscope treatment or suffered surgery, from January 2009 to March 2019, were included in this study. The patients were from 9 large Chinese third-grade class-A hospitals, among them, 8 were children-specialized hospitals, including Beijing Children's Hospital, Zhengzhou Children's Hospital, Xi'an Children's Hospital, Children's Hospital of Hebei Province, Anhui Provincial Children's Hospital, Jiangxi Provincial Children's Hospital, Children's Hospital of Nanjing Medical University, and Wuhan Children's Hospital, and 1 was a comprehensive hospital, the First Affiliated Hospital of Zhengzhou University.

\section{Data colletion}

Questionnaires were distributed online, medical records of the patients were retrospectively reviewed and special attention was paid on the age, gender, clinical manifestations, treatments, number and site of Buckyballs, and address of the patients' onset. Post-surgery follow-ups were through telephone and outpatient service.

\section{Statistic analysis}

All the data was analyzed using SPSS for Windows version 17.0. Normal distribution data was presented by (mean \pm standard deviation), non-normal distribution data was presented by median (first quartile, third quartile), and categorical variables were presented by frequencies and percentages.

\section{Results}

Alimentary tract Buckyball injury was diagnosed in 74 pediatric patients from the 9 large centers, including 8 children-specialized hospitals and 1 comprehensive hospital. Among the cases were 50 boys $(68 \%)$ and 24 girls (32\%), with an obvious gender prevalence that boys had higher incidence (Fig $1 \mathrm{~A})$. 
The age was $36.00(21.75,77.00)$ months, ranging from 7 months to 11 years old, and it showed two peaks, between one and three years old, and from six to eleven years old (Fig $1 \mathrm{~B}$ ). With the time progressed, more and more cases occurred and the annual case numbers showed sharp increase than it was in the past (Fig $1 \mathrm{C}$ ). Of all the provinces included, Hebei Province had the largest number of 23 cases, with Beijing and Henan Province came after with an equal number of 12 cases (Fig 1 D). All of the 74 patients had no definite diagnosed psychological disorders or allotriophagia. Only 3 of them had exact reasons for swallowing the balls, one neonatal boy was fed the balls by his elder cousin for amusement, another school age boy ate the balls on purpose to show his braveness, the rest one girl swallowed the balls as she regarded them as candies and was curious about the taste. The remaining 71 patients had no intension but accidental swallowed the balls while playing.

From onset to the emergency department time was $2.00(1.00,5.25)$ days, varied from 4 hours to 40 days, according to the symptoms. Twenty-one patients had no symptoms but had witness or told their parents about the swallowing and came to the hospital at an early onset time, other symptoms as abdominal pain, vomiting, fever, abdominal distension, fiercely crying, melena, and flatus and defecation stopped were in 40 (54.05\%), 36 (48.65\%), 11 (14.86\%), 3 (4.05\%), 3 (4.05\%), 2 (2.70\%), 1 (1.35\%) cases. Radiological examination could primarily display Buckyballs' numbers and location (Fig $2 \mathrm{~A}-\mathrm{C}$ ), which guided for better treatment. Sixteen patients tried gastroscope remove of the ingestion, nine successed while seven failed and changed to laparotomy. One patient attempted coloscope and also failed due to the powerful magnetic force of the balls. Six patients undertook laparoscopic operation and one changed to laparotomy. The rest 51 patients got laparotomy directly. During the exploratory surgery, gastrointestinal perforation (Fig $3 \mathrm{~A}$ ), ischemia and necrosis of gastrointestinal wall, abscess, ileus, fistula (Fig 3B-D) was seen in 38 (51.53\%), 14 (18.92\%), 7 (9.46\%), 6 (8.11\%), 2 (2.70\%), other 28 (37.84\%) patients had gastrointestinal mucosa erosion. The numbers of Buckyballs was $4(2,8)$, with a range from 1 to 39 .

During the follow-up period of $6(1,15)$ months, three patients had ileus after laparotomy surgery, and one of them suffered a second operation for relieving intestinal obstruction. The remaining 71 patients had no abdominal distension, adhesive intestinal obstruction, or tardive perforation incident. None of the 74 patients reported a second time swallowing of Buckyballs or other magnetic foreign bodies in the postsurgery course.

\section{Discussion}

Alimentary tract foreign body ingestion is common in pediatrics, especially in neonates and toddlers [3]. While among them, more than $80 \%$ need no intervention and the foreign body will pass out uneventfully, only $20 \%$ call for further attention, and less than $1 \%$ required surgery [4]. Treatments differ mainly based on the categories of foreign body, wait-and-see strategy could be used in the ordinary foreign body, such as coins, jewelries, and small toys, however $20 \%$ of patients had fish bones, jujube pits, batteries, or magnets ingestions need much more positive interventions. Early study revealed that from the year 2003 to 2009 , 38 cases of magnetic foreign body ingestion were identified, and 8 cases were multi magnets 
ingestion, and the number was still growing [5]. Another investigation in 2013 reported that during 2002 and 2011, there had been more than 22,000 pediatric magnetic foreign body ingestion cases in America, and a 5 fold increase was showed by comparing the first and the last 2 years [6]. Different from other foreign bodies, ingested magnets will attract each other across bowel loops, and cause gastrointestinal wall ischemia, pressure necrosis or perforation, especially when swallowed separately [7-8]. Other studies also described intestinal obstruction from internal hernia and volvulus of intestine, fistula formation[9], and hemorrhage when the mesenteric involved [9]. Midget $J$ reported a 20 months old boy who had such foreign body ingested, causing intestinal necrosis and abdominal sepsis, which led to the tragedy death [10-11]. Besides, Waters AM [12] reported another death case due to the hemorrhage from an esophagoaortic fistula induced by magnets ingestion.

Rare-earth magnet, consist of NdFeB (neodymium iron boron), a newly developed material, has a maximum magnetic energy product 5-10 times than the ordinary ferrite [11, 13-14], which means that the tiny rare-earth magnet could produce huge magnetic force. It was developed for the industrial manufacture, to decrease the volume of production and enlarge the poperties, such as electrical machinery, medical apparatus and instruments. Buckyballs, made of this kind of magnet, always consist of 216 magnets, are colorful in appearance and cheap to afford (Fig 4 A-B). But, it was developed for the crowd elder than 14 yeas of age for entertainment and pressure release (Fig $4 \mathrm{C}$-D). Besides, it could not only motivate the intelligence and creativity, but also ease the time mentally and physically through making wonderful creatures, both planar and triaxial, statically and dynamically. However, it also enjoys a rising popularity among children of all ages because of the toy abuse. Our investigation showed the peak was between one and three years old, and from six to eleven years old (Fig 1 B), consistent with De Roo AC's study in 2013 [15], which may be explained by the fact that toddlers explore the world with their mouths and may accidentally swallow the balls, and the school age children prove their braveness through the swallowing, as one case in this study. However 71 of the 74 patients were accidental, just consistent with the reports from De Roo AC [15] and the North American Society of Pediatric Gastroenterology, Hepatology and Nutrition (NASPGHAN) [3]. Other special reasons such as psychological disorders or allotriophagia $[5,14]$ were not fond in this study. The ingestion displayed an obvious male predominance at any age period (Fig $1 \mathrm{~A}$ ), which was in accordance with other reports [16]. This may attribute to the naughty and brave character, and the explorative spirit of boys.

Since Buckyballs were introduced to the market in $2009[13,17]$, the irreversible injuries increase than the past with the powerful magnetic force [18]. Unlike ordinary magnets, Buckyballs could attract each other even through six layers of intestinal walls [19], within an average distance of $3.5 \mathrm{~cm}$ [20], and strengthen will increase with multiple balls ingested [20]. Symptoms were not specific, and were decided by timing and location, but more than a half of the patients (40/74) showed abdominal pain, which was in accordance with Richard Sola Jr'sstudy [21] that abdominal pain was one risk factor for surgery. As in this study, the complications of ischemia and necrosis, gastrointestinal perforation (Fig 3 A), abscess, ileus, fistula (Fig 3 B-C) turned to be critical. Consequently, diagnosis and treatment algorithm were published by the NASPGHAN in 2012 [22], however, prevention is much more important. In the year 2014, Buckballs and high-powered magnets were mandatory recalled by the consumer product safety 
commission in the United States, and since the recall, a significant decrease was reported in multiple mini-magnet ingestion [23].

However, unlike America, our results (Fig 2C) discloses the truth that alimentary tract Buckyball injuries are still increasing sharply in China, with no trend to decline. It is the right time to take measures for the prevention, and some suggestions should be followed. Firstly, on the national administration level, the Buckyballs as well as other high-powered magnetic toys' production and trade should be stopped [14], and the publicity of serious health complications should be reinforce by all means of medias. Secondly, on the producer level, especially for the pediatric toy producers, national policy should be followed, the magnetic toy size should be enlarged, the material should be changed back to ordinary object that has low magnetic energy product [24], or magnetic force lowered to a flux index of 50kG2 [11], and the warning labels should be much more striking. Thirdly, on the consumer level, parents and caregivers should be realized about the potential risk of the toy $[10,17,24]$, and make children younger than 14 years old or have psychological disorders and allotriophagia away from it, strengthen the nursing, and educate children to well managed their toys before and after playing $[6,25]$. When evaluating their complaints of abdominal pain, specifically inquire about the ingestions should be took [14]. Children should be taught stop instigating others to swallow as well.

\section{Conclusion}

The issue of pediatric alimentary tract magnetic foreign body ingestion in China is still severe, multicenter study shows an increasing annual growth and severe gastrointestinal tract injuries. Treatments and managements are of great significance, while prevention is more important and time critical. Precautionary measures toward the issue should be taken from three levels, including the national administration, producer, and consumer, to decrease the morbidity.

\section{Acknowledgement}

The authors thank the entire staff of the Department of General Surgery, Beijing Children's Hospital, Zhengzhou Children's Hospital, Xi'an Children's Hospital, Children's Hospital of Hebei Province, Anhui Provincial Children's Hospital, Jiangxi Provincial Children's Hospital, Children's Hospital of Nanjing Medical University, and Wuhan Children's Hospital, and the First Affiliated Hospital of Zhengzhou University.

\section{Declarations}

\section{Funding}

This research did not receive any specific grant from funding agencies in the public, commercial, or notfor-profit sectors. 


\section{Availability of data and materials}

The data is available from the corresponding author on reasonable request.

\section{Authors' contributions}

Kai Wang conceived and designed the study, helped with the data collection, and drafted the initial manuscript. Dan Zhang, Xianling Li, Zengmeng Wang, Guangjun Hou, Xinjian Jia, Huizhong Niu, Shiqin Qi, Qingqiang Deng, Bin Jiang, Hongqiang Bian, and Heying Yang helped with the data collection. Yajun Chen conceived and designed the study, helped with data collection, and revised the manuscript. All authors read and approved the final manuscript.

\section{Ethics approval and consent to participate}

This study was reviewed and approved by the Ethics Committee of Beijing Children's Hospital. We retrospectively reported the data without providing any individual details.

\section{Consent for publication}

All the authors have approved the manuscript and agree for publication.

\section{Competing interest}

The authors declare that they have no competing interest.

\section{References}

1. Litovitz TL, Klein-Schwartz W, White S, et al. 2000 Annual report of the American Association of Poison Control Centers Toxic Exposure Surveillance System. Am J Emerg Med. 2001;19:337-95.

2. Gun F, Gunendi T, Kilic B, Celik A. Multiple magnet ingestion resulting in small bowel perforation: a case report. Ulus Travma Acil Cerrahi Derg. 2013;19:177-9.

3. Kramer RE, Lerner DG, Lin T, et al. Management of ingested foreign bodies in children: a clinical report of the NASPGHAN Endoscopy Committee. J Pediatr Gastroenterol Nutr. 2015;60:562-74.

4. Liu S, Li J, Lv Y. Gastrointestinal damage caused by swallowing multiple magnets. Front Med. 2012;6:280-7. 
5. Tavarez MM, Saladino RA, Gaines BA, et al. Prevalence, clinical features and management of pediatric magnetic foreign body ingestions. J Emerg Med. 2013;44:261-8.

6. Silverman JA, Brown JC, Willis MM, et al. Increase in pediatric magnet-related foreign bodies requiring emergency care. Ann Emerg Med. 2013;62:604-8.

7. Baines H, Saenz NC, Dory C, et al. Magnet-associated intestinal perforation results in a new institutional policy of ferromagnetic screening prior to MRI. Pediatr Radiol. 2012;42:1506-9.

8. Agbo C, Lee L, Chiang V, et al. Magnet-related injury rates in children: a single hospital experience. J Pediatr Gastroenterol Nutr. 2013;257:14-7.

9. Bauman B, McEachron K, Goldman D, et al. Emergency Management of the Ingested Magnet: An Algorithmic Approach. Pediatr Emerg Care. 2017;1:1-4.

10. Centers for Disease Control and Prevention: Gastrointestinal injuries from magnet ingestion in children-United States, 2003-2006. MMWR Morb Mortal Wkly Rep. 2006;55:1296-300.

11. Alfonzo MJ, Baum CR. Magnetic Foreign Body Ingestions. Pediatr Emerg Care. 2016;32:698-702.

12. Waters AM, Teitelbaum DH, Thorne $\mathrm{V}$, et al. Surgical management and morbidity of pediatric magnet ingestions. J Surg Res. 2015;199:137-40.

13. Robey TE, Kaimakliotis HZ, Hittelman AB, et al. An unusual destination for magnetic foreign bodies. Pediatr Emerg Care. 2014;30:643-5.

14. Brown JC, Otjen JP, Drugas GT. Pediatric magnet ingestions: the dark side of the force. Am J Surg. 2014;207:754-9.

15. De Roo AC, Thompson MC, Chounthirath $\mathrm{T}$, et al. Rare-earth magnet ingestion-related injuries among children, 2000-2012. Clin Pediatr. 2013;52:1006-13.

16. Abbas Ml, Oliva-Hemker M, Choi J, Lustik M, et al. Magnet ingestions in children presenting to US emergency departments, 2002-2011. J Pediatr Gastroenterol Nutr. 2013;57:18-22.

17. Brown JC, Baik FM, Ou HC, et al. Upper aerodigestive magnetic foreign bodies in children. Laryngoscope. 2014;124:1481-5.

18. Strickland M, Rosenfield D, Fecteau A. Magnetic foreign body injuries: a large pediatric hospital experience. J Pediatr. 2014;165:332-5.

19. Mandhan P, Alsalihi M, Mammoo S, et al. Troubling toys: rare-Earth magnet ingestion in children causing bowel perforations. Case Rep Pediatr. 2014; doi: 10.1155/2014/908730. 
20. Tsai J, Shaul DB, Sydorak RM, et al. Ingestion of magnetic toys: report of serious complications requiring surgical intervention and a proposed management algorithm. Perm J. 2013;17:11-4.

21. Sola RJ, Rosenfeld EH, Yu YR, et al. Magnet foreign body ingestion: rare occurrence but big consequences. J Pediatr Surg. 2018;53:1815-9.

22. Hussain SZ, Bousvaros A, Gilger M, et al. Management of ingested magnets in children. J Pediatr Gastroenterol Nutr. 2012;55:239-42.

23. Rosenfield D, Strickland M, Hepburn CM. After the Recall: Reexamining Multiple Magnet Ingestion at a Large Pediatric Hospital. J Pediatr. 2017;186:78-81.

24. Hodges NL, Denny SA, Smith GA. Rare-Earth Magnet Ingestion-Related Injuries in the Pediatric Population: A Review. Am J Lifestyle Med. 2017;11:259-63.

25. Liu S, Li J, Lv Y. Gastrointestinal damage caused by swallowing multiple magnets. Front Med. 2012;6:280-7.

\section{Figures}


Gender distributioin

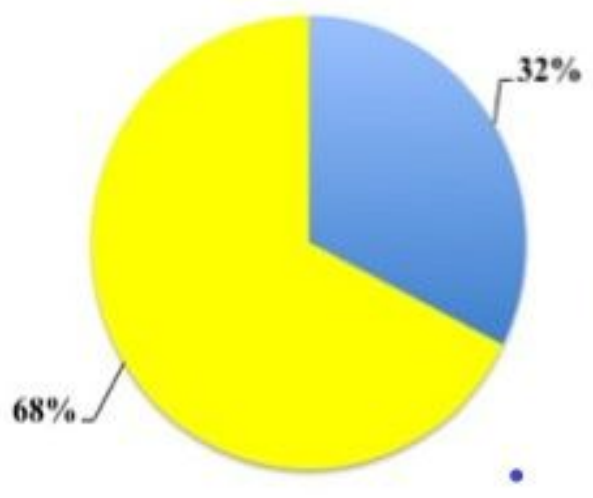

ifemal male
Age distribution

Number of cares

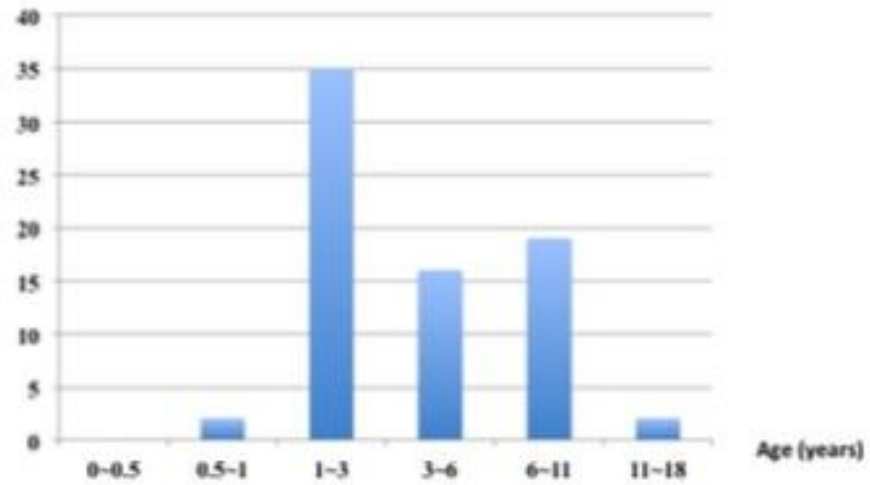

D

Year distribution

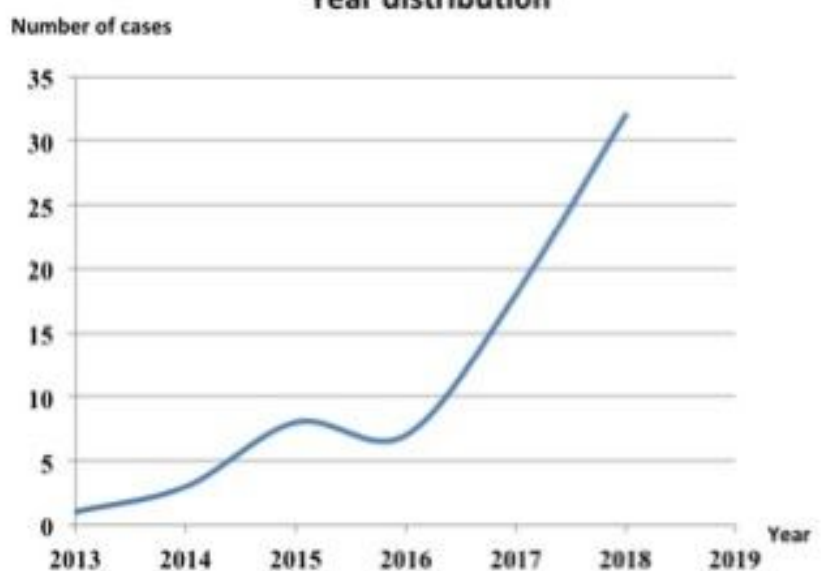

Number of cases

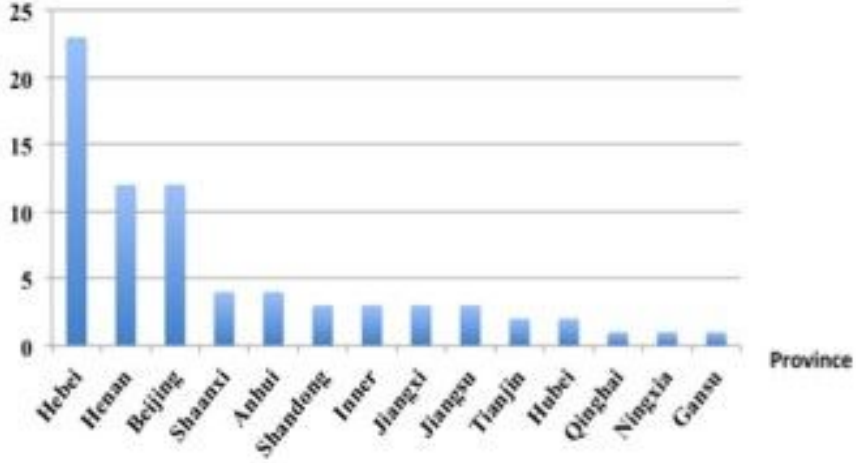

Figure 1

Results showed the gender (A), age (B), year (C) and area (D) distribution of Buckyball injuries 

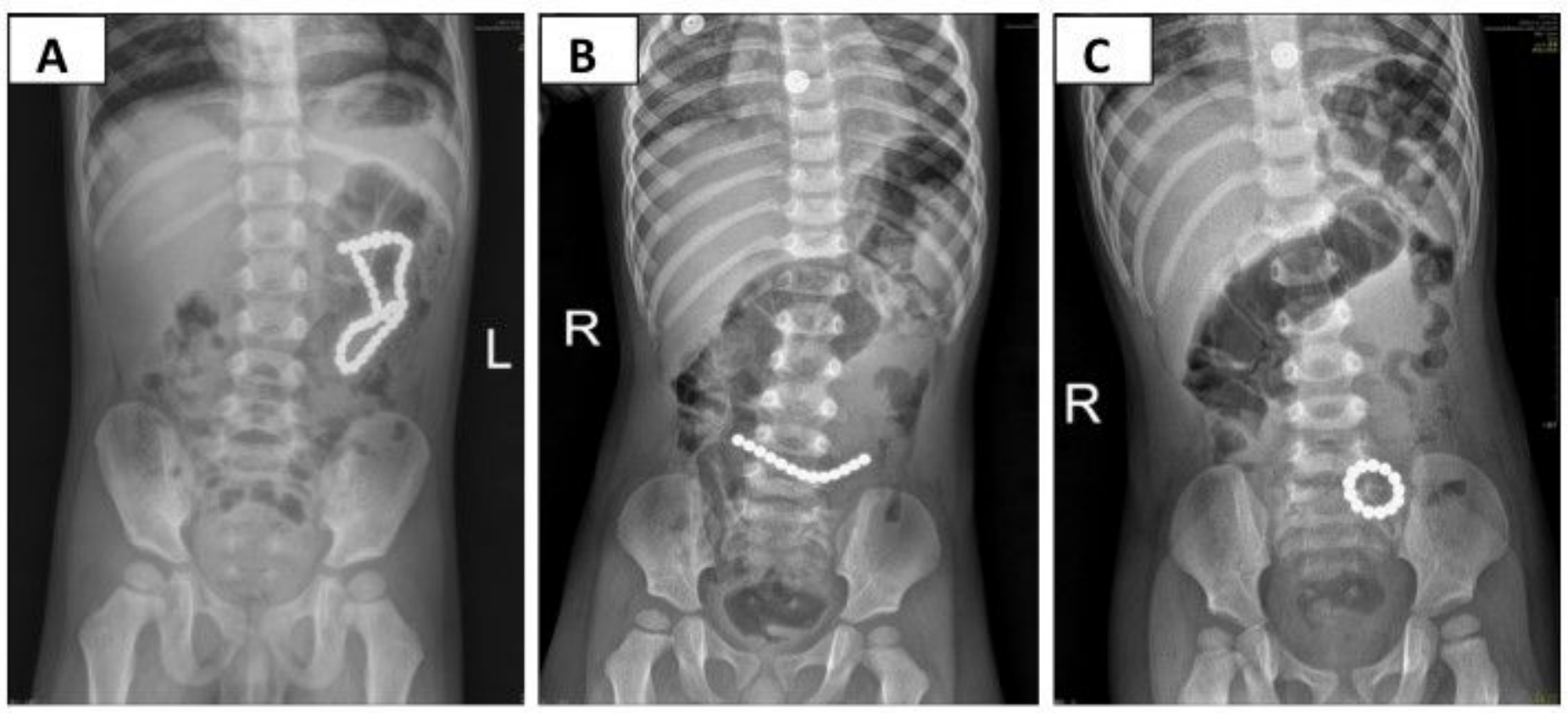

Figure 2

Radiology results of Buckyballs ingested. The maximum of 39 Buckyballs ingested (A). Another patient ingested 13 Buckyballs, which showed line type on the first day (B), and turned to annular type on the following day (C) 

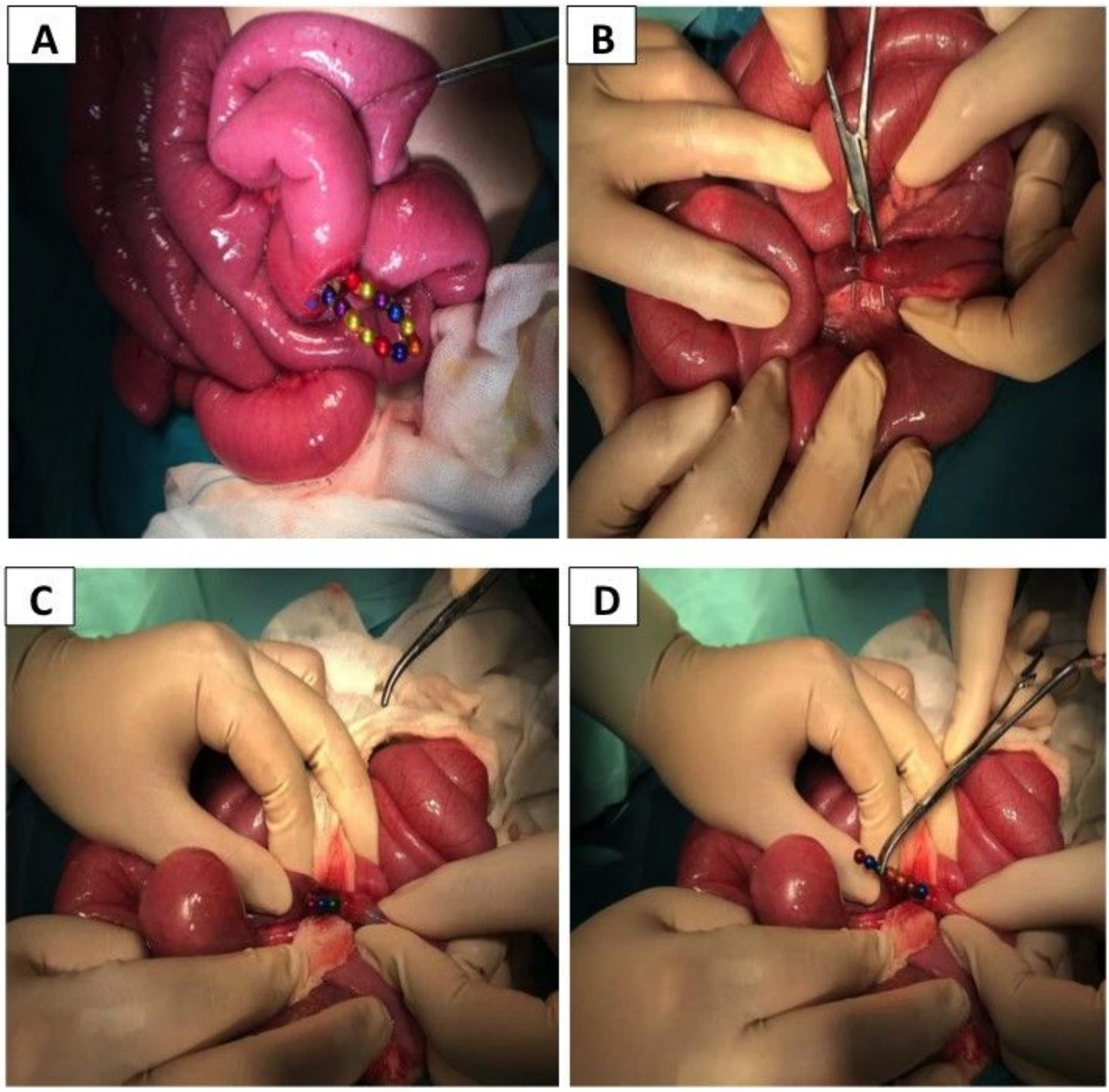

Figure 3

Exploration results during surgery. Buckyballs caused intestinal perforation (A) and fistula (B-D) 


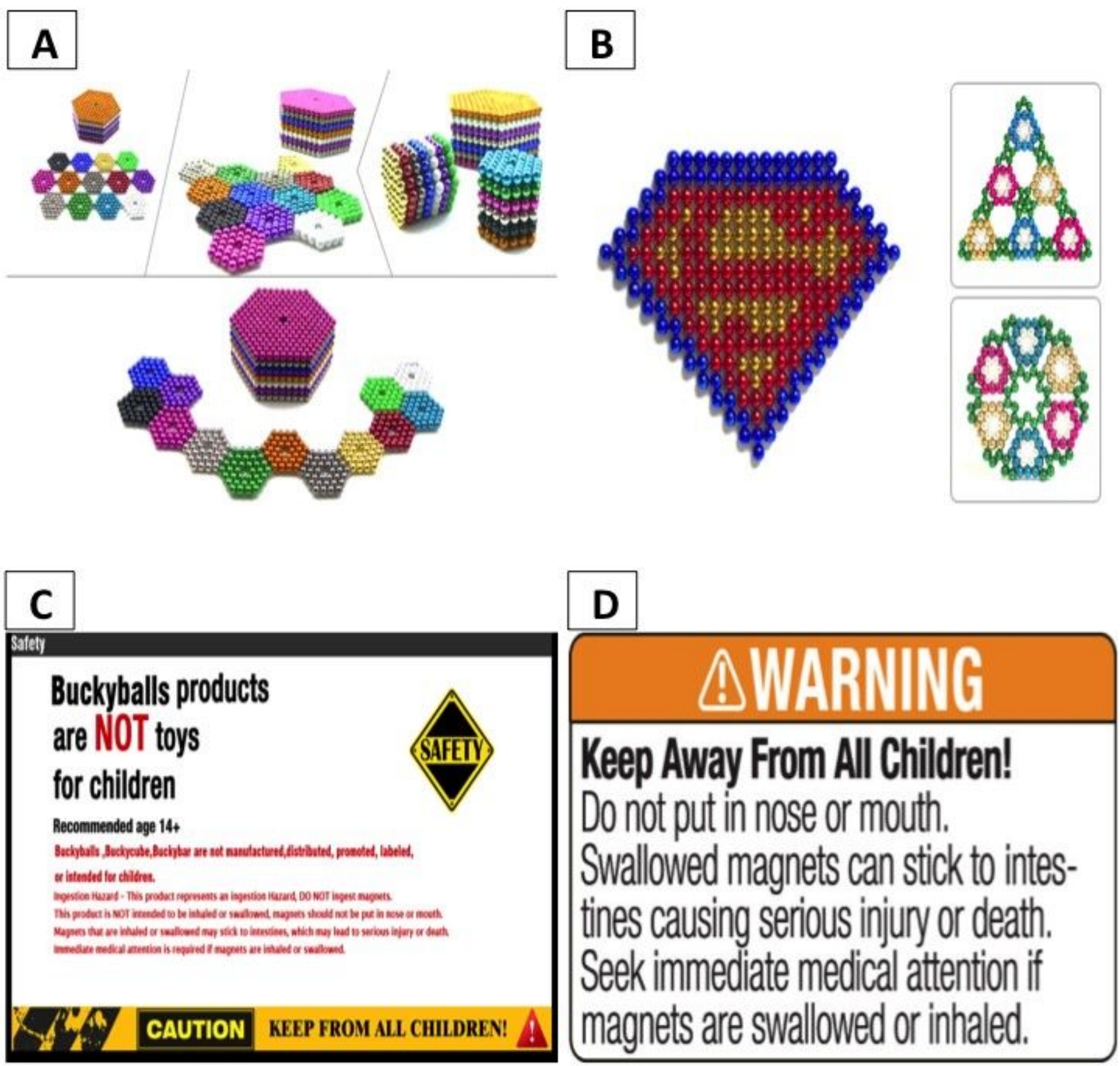

Figure 4

Buckyballs with different shapes and colors, and the warning labels that showed the accessible age was upon 14 years old 